Meta

Journal des traducteurs

Translators' Journal

\title{
Synonymie, polysémie et classes d'objets
}

\section{Gaston Gross et André Clas}

Volume 42, numéro 1, mars 1997

Lexicologie et terminologie

URI : https://id.erudit.org/iderudit/002977ar

DOI : https://doi.org/10.7202/002977ar

Aller au sommaire du numéro

Éditeur(s)

Les Presses de l'Université de Montréal

ISSN

0026-0452 (imprimé)

1492-1421 (numérique)

Découvrir la revue

Citer cet article

Gross, G. \& Clas, A. (1997). Synonymie, polysémie et classes d'objets. Meta, 42(1),

147-154. https://doi.org/10.7202/002977ar

\section{Résumé de l'article}

La désambiguïsation de la polysémie et la différenciation des synonymes s'appuient sur une analyse sémasyntaxique. Grâce à la création de classes d'objets, il est possible de raffiner la description des mots et de les présenter de telle façon qu'un traitement automatique d'analyse et de génération automatique soient possibles. 


\title{
SYNONYMIE, POLYSÉMIE ET CLASSES D'OBJETS*
}

\author{
Gaston Gross et André Clas \\ Laboratoire de Linguistique informatique, Université de Paris XIII, France et \\ GRESLET, Département de linguistique, Université de Montréal, Canada
}

\begin{abstract}
Résumé
La désambiguïsation de la polysémie et la différenciation des synonymes s'appuient sur une analyse sémasyntaxique. Grâce à la création de classes d'objets, il est possible de raffiner la description des mots et de les présenter de telle façon qu'un traitement automatique d'analyse et de génération automatique soient possibles.
\end{abstract}

\begin{abstract}
Disambiguation of polysemy and differentiation of synonyms depend on semasyntactic analysis. By creating classes of objects, it is possible to refine word descriptions in such a way as to facilitate computerized analysis and generation.
\end{abstract}

\section{GÉNÉRALITÉS}

Il est quelque peu banal de constater que les langues naturelles se servent souvent des mêmes sons pour désigner des sens différents, c'est-à-dire qu'il existe dans les langues des éléments lexicaux qui sont polysémiques, et des sons différents pour désigner des sens identiques, ou au moins semblables, c'est-à-dire que les langues connaissent des éléments lexicaux qui sont synonymiques. On voit ainsi, et ce n'est pas nouveau, que toute étude sémantique passe obligatoirement par la désambiguïsation de la polysémie (délimiter les différentes significations d'un mot) et la différenciation de la signification des synonymes (déterminer la signification identique, presque identique ou simplement proche des mots ayant le «même» sens).

Si l'on admet avec J. Apresjan (1963) que toute différence sémantique ne se manifeste pas dans une différence syntaxique, mais qu'à chaque différence syntaxique correspond une différence sémantique essentielle, on aboutit à une analyse distributionnelle de la signification lexicale. En d'autres mots, chaque sens d'une unité lexicale est déterminé par ses propriétés grammaticales ou, d'une façon plus large, il existe un certain isomorphisme entre la sémantique et la grammaire. C'est bien ce qu'affirmait déjà Z. Harris qui montre que les mots sont déterminés par leurs propriétés grammaticales: les possibilités syntaxiques correspondent aux différents sens. Cependant, la proposition distributionnelle de Z. Harris se complète par le regroupement des éléments du lexique sous une représentation plus généralisée.

\section{DÉCOMPOSITION PHRASÉOLOGIQUE}

La phrase canonique se décompose, selon la plupart des grammairiens, en opérateur et arguments. Il s'agit ici d'utiliser la technique du calcul des prédicats pour représenter la structure de la phrase simple qui affirme que les phrases sont formées par des termes (au sens technique) qui représentent des noms individuels et des prédicats. Tout opérateur, c'est-à-dire tout élément qui sert à constituer une phrase, est muni par conséquent d'arguments (un sujet et un ou plusieurs compléments) et se détermine par la nature de 
ceux-ci. On en exclut les noms qui ne peuvent pas avoir d'arguments, tels les noms concrets (objets, animaux, végétaux...).

On détermine les prédicats en fonction du nombre d'arguments. Rappelons qu'il existe non seulement des prédicats verbaux, mais aussi des prédicats nominaux. En effet, un grand nombre de substantifs ont des arguments. Ces substantifs opérateurs ou prédicatifs (noms d'actions, noms d'états, noms d'événements) ont en effet toujours un sujet, et la plupart du temps un ou plusieurs compléments.

\section{LES TRAITS SYNTAXIQUES}

Une classification qui subdivise les noms en sous-ensembles grâce à des traits comme humain, concret, abstrait, locatif, animé, etc., permet de préciser les «qualités» de l'opérateur. Ces mêmes traits permettent également de distinguer différents emplois d'opérateurs. Ainsi, on oppose, par exemple, Paul chante et La bouilloire chante. On peut donc distinguer deux emplois de chanter, dans le premier cas, le sujet a le trait humain, alors que dans le deuxième cas, le sujet a le trait concret. Les traits syntaxiques qui permettent de telles descriptions sont: humain, animal, végétal, inanimé concret, locatif, temps, action, état, événement.

Mais il est facile de montrer que les traits ainsi dégagés ne permettent pas une désambiguïsation suffisante dans de très nombreux cas. Ainsi, si l'on veut décrire le verbe tailler, on ne peut se contenter des traits humain et concret, car si la phrase Paul taille le crayon est tout à fait acceptable, il n'en sera pas de même pour *Paul taille la lampe ou *Paul taille l'encrier. Il s'agit donc d'établir d'autres indications qui permettent de construire des phrases acceptables et rien que celles-ci. Comme une énumération de tous les substantifs qui constituent les arguments de l'opérateur est une tâche de très longue haleine, car la liste de ces éléments est dans de nombreux cas presque illimitée et encore répétitive pour de nombreux arguments avec des opérateurs différents, il faut absolument généraliser la représentation et créer un sous-ensemble de traits que l'on peut appeler classes d'objets. Ainsi, pour reprendre l'exemple du verbe tailler, on peut désambiguïser les phrases suivantes qui s'analysent également par les traits humain et concret, en spécifiant les classes d'objets puisées dans le réel: Paul taille un cerisier et Paul taille une veste. Comme l'affirme John Lyons (1995: 218): «although natural languages vary considerably as to what they grammaticalize (or lexicalize), there is, in all natural languages, some degree of congruence between semantic (or ontological) categories and certain grammatical categories». On précisera donc que «cerisier» relève de la classe d'objets <arbre> et que «veste» appartient à la classe d'objets <vêtement>. Bien plus, on pourrait même spécifier que le sujet n'est pas n'importe quel humain, mais un substantif qui appartient à une classe d'objets comme <profession>, par exemple. On écrira alors : L'émondeur (l'élagueur) taille le cerisier et Le tailleur (la couturière) taille la veste.

\section{LES TRAITS SYNTACTICO-SÉMANTIQUES}

Comme nous venons de le voir, si l'on décrit simplement les arguments à l'aide de traits minimaux, tels que les traits humain ou concret, dans une perspective de traitement automatique, il est évident que l'on pourrait générer des milliards de phrases en français dont un très grand nombre, pour ne pas dire la plupart, seraient fausses. La raison en est toute simple: tous les concrets de la langue ne peuvent fonctionner avec le même opérateur.

Il s'agit donc de rassembler en des ensembles les substantifs qui sont sémantiquement et syntaxiquement homogènes et qui ont comme fonction de décrire avec la précision voulue les emplois d'opérateurs. La démarche part de la langue et non d'arbres prédéterminés qui découpent l'univers selon la représentation mentale du concepteur. 
La démarche préconisée n'a donc rien de commun avec celle que l'on trouve, par exemple, dans le dictionnaire électronique du système de traduction automatique SYSTRAN où les indications sémantiques sont présentées sous forme d'arbre de dérivations sémantiques comprenant 800 traits taxonomisés en six arborescences: Choses, Actions, États, Qualités, Lieux, Positions relatives. Chaque mot doit appartenir à l'une de ces arborescences. Dans chaque taxinomie, il y a un nœud pour chaque division et un code qui ne couvre qu'un seul concept. Un terme reçoit donc automatiquement tous les codes afférents aux nœuds supérieurs jusqu'au taxon de tête. Si un seul code ne convient pas au terme en question, on ne peut lui attribuer le code inférieur, à moins de lui donner un «stoppeur» et de lui adjoindre le code d'un des cinq autres taxons. Il n'a rien de commun non plus avec le dictionnaire électronique en cours de réalisation au Japon (An Electronic Dictionary for Knowledge Information Processing) qui prévoit, pour la traduction automatique et un système de compréhension de la parole, la rédaction de deux dictionnaires électroniques fondamentaux : un dictionnaire principal qui sera formé de deux dictionnaires. Chaque dictionnaire comprend d'autres dictionnaires. Le premier dictionnaire est composé d'un dictionnaire général, japonais et anglais, de 200000 mots, et d'un dictionnaire spécialisé, japonais et anglais, de 100000 mots du domaine du traitement de l'informatique. Le deuxième dictionnaire comprend une description conceptuelle et une classification conceptuelle des entrées des deux autres dictionnaires. On peut représenter ces dictionnaires de la façon suivante:

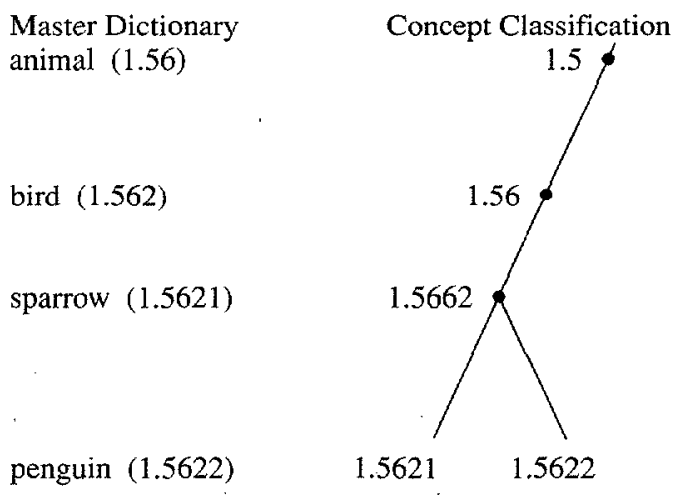

Concept Description
1.562
leg:two
breeding:oviparous
movement:flying
1.5621
color:brown
habitat:human habitat
1.5622
color:black,white
habitat:colder coast of
Southern Hemispere
movement:walking

Comme il s'agit dans les classes d'objets de représenter la description de la langue, on voit facilement que les traits que l'on trouve habituellement en vue du codage des noms dans les dictionnaires électroniques, comme humain, concret, abstrait, sont insuffisants pour décrire la langue en vue d'un décodage ou d'un encodage automatique. Ainsi, le trait humain ne permet de décrire que cette catégorie de substantifs alors qu'ils ont en commun un certain nombre de prédicats avec des substantifs décrivant des animaux, par exemple. L'usage de l'hyperonyme animé ne résout pas non plus le problème, car comment ranger les végétaux, qui sont des concrets, mais qui partagent, par exemple, les prédicats avec les humains et les animaux comme naître, croître, péricliter, mourir. Il faut donc ajouter ces deux traits à la liste initiale.

Quand on examine des mots comme route, stade, plaine, pays, ville, etc., on constate que l'addition d'un trait comme locatif permet de mieux coder ce type de mots. On remarque également que de nombreuses prépositions régissent à la fois les compléments de temps et de lieu, mais qu'il existe cependant des différences notables, et la création d'un trait comme temps donne une meilleure description de ces substantifs. 
Si l'on analyse des substantifs qui ne sont pas des prédicats, c'est-à-dire les substantifs-arguments, que l'on peut coder comme concrets, on voit que cet étiquetage seul ne permet pas de résoudre le fonctionnement de l'ensemble des substantifs de cette catégorie. Il en est de même pour les substantifs abstraits qui eux, par contre, sont des prédicats nominaux. Un substantif comme explosion est codé comme abstrait au même titre que gentillesse, par exemple. Or, explosion ne peut être étiqueté que comme abstrait. Les deux mots, en revanche, affichent un comportement syntaxique différent (emploi avec les verbes être de et avoir pour gentillesse et utilisation de avoir lieu et de se produire pour explosion). Les verbes avoir lieu, se produire, etc., actualisent des prédicats d'événements.

D'après tout ce qui précède, on voit que les propriétés syntaxiques exposées permettent la définition de huit traits syntactico-sémantiques, soit: humain, animal, végétal, inanimé concret, inanimé abstrait, locatif, temps, événement (les abréviations sont: hum, ani, vég, inc, ina, loc, tem, évé). Le classement des substantifs se fait, dans un premier temps, à l'aide de ces huit traits. Ainsi, le mot cuisinière, dans des phrases comme Je vous présente la cuisinière et $J$ 'achète une cuisinière émaillée montrent que le substantif «cuisinière» doit être codé comme humain et comme concret. Par exemple, un substantif comme réflexion sera décrit de la façon suivante:

réflexion / NO:hum / NI :sur /W:faire / V:réfléchir / A:réfléchi

où N0 désigne le sujet, $\mathrm{N} 1$ un premier objet, $\mathrm{W}$ le verbe support, $\mathrm{V}$ le verbe de même sens et de même racinc que le nom, A l'adjectif qui est relié au nom prédicatif. Pour informatiser convenablement cette description, on utilise cette même grille pour tous les substantifs. Si le substantif a un deuxième objet, on le notera par N2, sinon la place reste vide, tout comme d'ailleurs pour les prédicats nominaux qui n'ont pas de verbe (de même racine et de même sens que le substantif) ou pas d'adjectif (de même racine et de même sens que le substantif); en plus, si le verbe support a des variantes, on les indiquera en les séparant par une virgule, étant donné qu'il n'y a pas création d'un nouveau prédicat, mais simplement une variation, en général, d'un niveau de langue. La grille se lira donc comme suit:

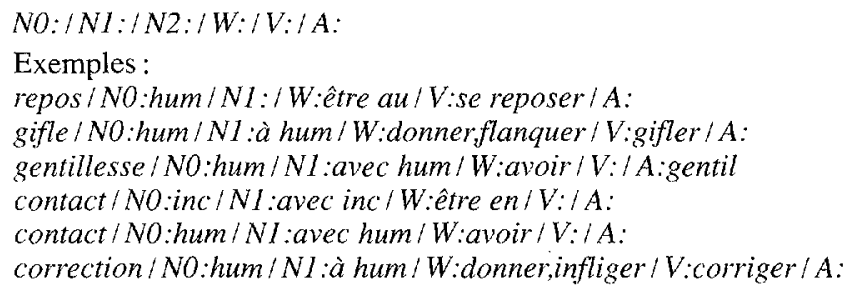

\section{LES CLASSES D'OBJETS}

La description ainsi fournie n'est cependant pas suffisante pour coder ou décoder automatiquement un texte. On se rend rapidement compte que le trait, par exemple, concret qui s'applique à un ensemble de termes, ensemble que nous appelons prédicats généraux, ne suffit pas à déterminer tous les termes qui relèvent de cet ensemble. En analysant, par exemple, tous les emplois possibles de porter, on s'aperçoit facilement, notamment lorsqu'on compare avec d'autres langues, que l'analyse des phrases suivantes:

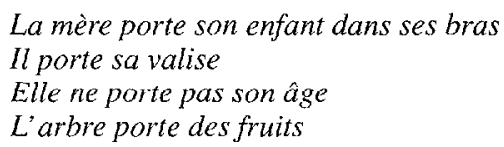




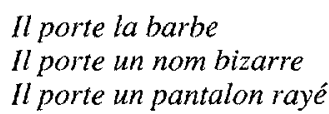

ne se laisse pas simplement résoudre avec les traits humain, concret et abstrait. En effet, si certains traits permettent de distinguer certaines phrases, il n'en reste pas moins que la recherche de l'équivalent en anglais n'autorise pas la déduction que celui-ci est toujours le même. Ainsi, si porter avec un inanimé concret se traduit par to carry et si même un humain se traduit par le même verbe, on constate que d'autres inanimés concrets correspondent à d'autres verbes. On aura donc:

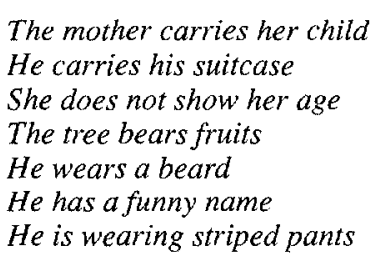

On se voit donc obligé de préciser les traits, en plus de créer des classes sémantiques plus fines que nous appelons classes d'objets. On peut alors affirmer qu'un substantif est caractérisé par deux descriptions:

- les prédicats généraux qui caractérisent tous les termes qui ont le même trait syntactico-sémantique, par exemple les concrets;

- les prédicats appropriés qui caractérisent les divers éléments d'une classe d'objets, par exemple les vêtements.

Ainsi, le substantif pantalon, décrit par le trait concret, pourra accepter tous les prédicats qui se rapportent à cette catégorie, soit être (nom de couleur), coûter (quantification unité de prix), mesurer (quantification unité de mesure), peser (quantification unité de poids), etc. En plus, pantalon a en commun avec l'ensemble des termes de vêtements (manteau, chemise...) des prédicats appropriés à cette classe d'objets comme mettre, porter, enlever, enfiler, repasser, etc.

Pour pouvoir décrire adéquatement la langue, nous croyons qu'il est indispensable, si l'on veut obtenir une description valable qui permette de résoudre les problèmes de traduction automatique, par exemple, de lister les fonctions variables des prédicats et des arguments. Comme on sait que les langues diffèrent largement par les prédicats appropriés, il est indispensable de sous-catégoriser les traits syntactico-sémantiques en autant de classes d'objets qu'il est nécessaire pour obtenir un traitement informatique efficace. On établira ainsi la syntaxe de chaque classe d'objets. Si l'on dresse la liste de tous les membres d'une classe d'objets et si l'on a la syntaxe de cette même classe, on est en mesure de décoder et d'encoder automatiquement toutes les constructions possibles. C'est bien sûr un travail d'analyse et de description long et minutieux, mais il nous semble tout à fait faisable, et ce n'est qu'à ce prix que l'on pourra faire progresser le traitement automatique!

À titre indicatif, on peut établir la liste des classes d'objets pour les substantifs qui ont le trait humain:

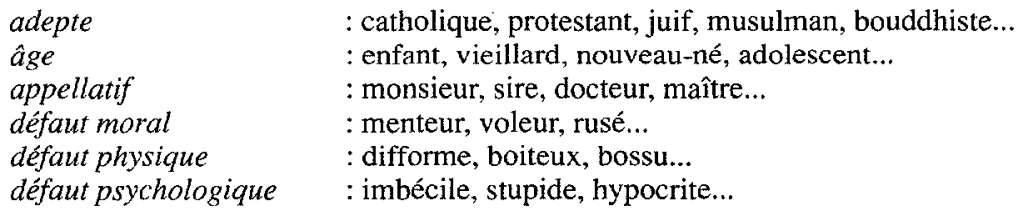




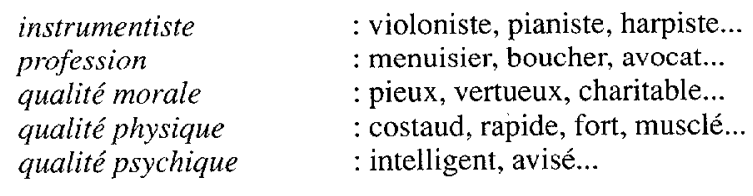

La mise au point des classes d'objets ne se fonde pas uniquement sur l'intuition, c'est-à-dire sur un regroupement de substantifs sémantiquement homogènes, mais plus profondément sur le comportement syntaxique des prédicats appropriés. La description des substantifs comprend trois niveaux descriptifs:

- niveau 1 : classement des substantifs en fonction de leurs traits syntaxiques. Tous les opérateurs qui leur sont spécifiques seront ainsi déterminés;

- niveau 2: sous-catégorisation des traits en classes d'objets en fonction de la description des relations opérateurs-arguments;

- niveau 3 : recensement pour chacune des classes des prédicats appropriés, c'està-dire établissement des syntaxes locales.

$\mathrm{Si}$, à titre d'illustration, on décrit le terme autobus, on peut fixer, en conformité avec le schéma ci-dessus, les étapes suivantes:

a) attribution du trait inanimé concret. Le terme acquiert du même coup toutes les propriétés qui définissent les substantifs concrets, plus spécifiquement:

- il peut être argument-sujet avec les verbes comme avoir (une couleur), avoir (un poids), mesurer (unité de mesure), coûter (unité de prix), se trouver (à tel endroit);

- il peut être objet de verbes comme voir, apercevoir, peindre en, etc. En fait, il hérite, comme tous les autres substantifs de la classe, toutes les propriétés du trait inanimé;

- il se soumet aux propriétés syntaxiques des concrets, c'est-à-dire utilisation des pronoms en et $y$ (par opposition aux pronoms de lui ou à lui appartenant aux humains);

- il prend qu' est-ce que au lieu de qui dans les interrogations, etc.

b) inscription dans la classe d'objets <moyens de transports routiers en commun>;

c) description des environnements potentiels du terme. Rappelons que cette même description sera valable pour tous les éléments de cette même classe d'objets. Les prédicats qui concernent le terme en question sont ceux des $\langle$ transports routiers $\rangle$, c'est-à-dire qui acceptent les verbes comme démarrer, rouler, s'arrêter au feu rouge, être en nième vitesse, prendre un virage, dépasser, doubler, stationner, faire un tête à queue, etc. - En plus, le terme sera décrit par les prédicats des <moyens de transport en commun > qui sont marqués par d'autres propriétés syntaxiques qui indiquent l'opposition par rapport aux moyens de transport individuels:

- indications de départ et de destination l'autobus de Montréal mais * la voiture de Montréal

- spécification d'horaire l'autobus de 7 h30 mais *la voiture de 7 h30

- prédication appropriée rater, manquer, louper son autobus mais ?rater la voiture

- synonymie particulière prendre (emprunter) un autobus mais prendre (*emprunter) la voiture. 


\section{ILLUSTRATION}

Si 1'on choisit un peu au hasard un adjectif, par exemple abominable, et si l'on consulte les dictionnaires de synonymes, on trouve les entrées suivantes:

Dictionnaire $1:$ Abominable $1 \rightarrow$ Haïssable. $2 \rightarrow$ Détestable.

Dictionnaire 2 : abominable 1. Cet abominable individu a commis un crime abominable; adj. dont il est difficile d'enumérer et surtout de classer tous les syn.; citons affreux, atroce, détestable, horrible, monstrueux, épouvantable, exécrable. -2 Il fait un temps abominable: sorte de superlatif de mauvais; on pourrait ordonner, par ordre de degré croissant d'intensité : mauvais, détestable, abominable, effroyable, exécrable, horrible, épouvantable, désastreux, catastrophique.

Dans le dictionnaire 1, les renvois donnent pour haissable: adieux, antipathique, détestable, exécrable et abominable (avec des explications et des citations), et pour détestable: abominable, exécrable, odieux, maudit, damné et sacré, toujours avec des explications de nuances de sens et des exemples.

On voit que de tels dictionnaires ne sont évidemment pas faits pour un traitement électronique: la machine ne peut remplacer l'intuition indispensable du lecteur humain. Par contre, l'analyse synonymique que nous proposons permet à un système informatique de s'y retrouver. L'analyse classe tout d'abord l'entrée abominable dans environ onze emplois différents. On obtient ainsi le tableau suivant (où les éléments entre crochets indiquent les classes d'objets, les éléments entre parenthèses des domaines d'emploi, No représente le sujet, $\mathrm{N} 1$ le complément):

abominable 1 : N0 ina(nimé), <acte> (crime), Syn: terrible, horrible, monstrueux, atroce ;

abominable 2 : No ina, <texte, propos, pensée>, Syn: détestable, exécrable, abject; Ant(onyme) : beau, intéressant;

abominable 3 : N0 hum, N1 dans Poss <action>, Syn : exécrable, détestable, odieux, monstrueux; Ant: aimable, adorable;

abominable 4 : No hum, Syn: laid, repoussant ;

abominable $5 \quad$ : N0 inc $<$ npc $>$ (visage), Syn : laid, repoussant; Ant : beau, séduisant;

abominable 6 : N0 évé(nement) (accident), Syn : épouvantable, horrible;

abominable 7 : N0 ina (odeur), Syn : exécrable, infect;

abominable 8 : No ina <Nmétéo $>$ (temps), Syn: mauvais, épouvantable, affreux, exécrable; Ant : beau, agréable ;

abominable 9 : N0 loc, Syn : détestable, exécrable; Ant : agréable;

abominable 10 : N0 loc <voie>, Syn : difficile; Ant: facile, agréable;

abominable 11 : N0 inc<objet>, ina <œuvre>, Syn: laid, affreux; Ant: beau, magnifique.

\section{CONCLUSION}

La description proposée permet des applications multiples tant du point de vue du décodage que de l'encodage automatiques. La traduction automatique serait une première application puisque la catégorisation par classes d'objets permet de résoudre l'ambiguité créée par la polysémie et la formulation adéquate des équivalents grâce aux syntaxes locales qui y sont attachées. Une autre application serait la génération automatique de textes, tout comme, d'ailleurs, la documentation automatique puisqu'il serait possible 
d'interroger sur les substantifs ayant tel ou tel trait particulier, de chercher la liste des objets appartenant à la même classe, d'établir la liste des opérateurs appropriés qui s'appliquent à telle ou telle classe. On peut aussi facilement entrevoir les possibilités pour une nouvelle pégagogie pour l'enseignement de la langue maternelle ou des langues étrangères. Sans oublier des applications «stylistiques» dans le cadre d'un «dictionnaire de désambiguïsation synonymique».

Note

* Recherche effectuée dans le cadre de la Coopération scientifique et technologique entre le Québec et la France, Secteur: Ingénieries linguistique et de la connaissance.

\section{RÉFÉRENCES}

A.PRESJAN, Jurij (1963) : «Analyse distributionnelle des significations et champs sémantiques structurés», Langages, 1, mars 1963, Paris, Didier-Larousse, pp. 44-74.

DUCHACEK, Otto (1964): «Différents types de synonymes», Orbis, XIII-1, pp. 35-49.

GROSS, Gaston (1981) : «Les bases empiriques de la notion de prédicat sémantique», Langages, 3, Paris, Larousse.

GROSS, Gaston (1989) : Les constructions converses du français, Genève, Droz.

GROSS, Gaston (1992): «Forme d'un dictionnaire électronique», André Clas et Hayssam Safar (dir.) : L'environnement traductionnel, la station de travail du traducteur de l'an 200I, Actes du Colloque de Mons, Sillery, Presses de l'Université du Québec et AUPLEF-UREF, pp. 255-271.

GROSS, Gaston (1994) : «Classes d'objets et description des verbes», Langages, 115, Paris, Larousse.

GROSS, Gaston (1995) : «Connecteurs et traitement automatique», André Clas et Pierrette Bouillon (dir.) : TA-TAO : recherches de pointe et applications immédiates, Actes du colloque de Montréal, Beyrouth, FMA et AUPELF-UREF, pp. 287-305.

LYONS, John (1995) : Linguistic Semantics, Cambridge, Cambridge University Press. 